\title{
Multidimensional (co)evolutionary stability
}

\author{
Flo Débarre
}

\author{
@flodebarre \\ University of Exeter, UK.
}

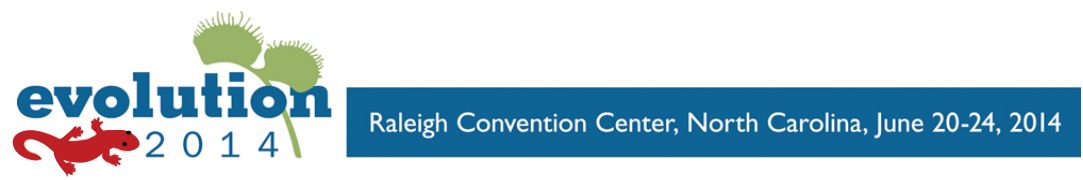




\title{
Multidimensional (co)evolutionary stability
}

\author{
Flo Débarre
}

\author{
3)flodebarre \\ University of Exeter, UK.
}

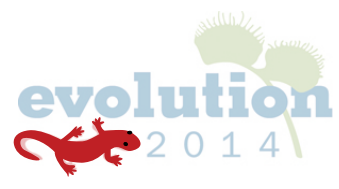

Raleigh Convention Center, North Carolina, June 20-24, 2014 


\title{
Multidimensional (co)evolutionary stability
}

\author{
Flo Débarre
}

@flodebarre

University of Exeter, UK.

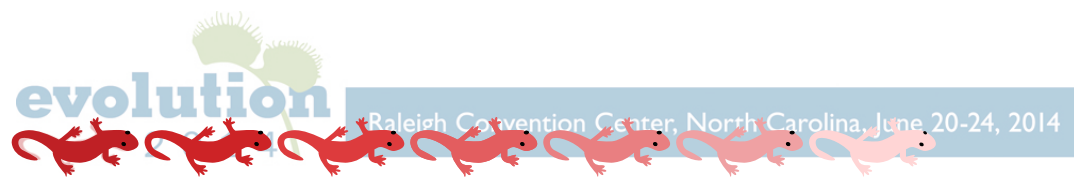




\title{
Multidimensional (co)evolutionary stability
}

\author{
Flo Débarre
}

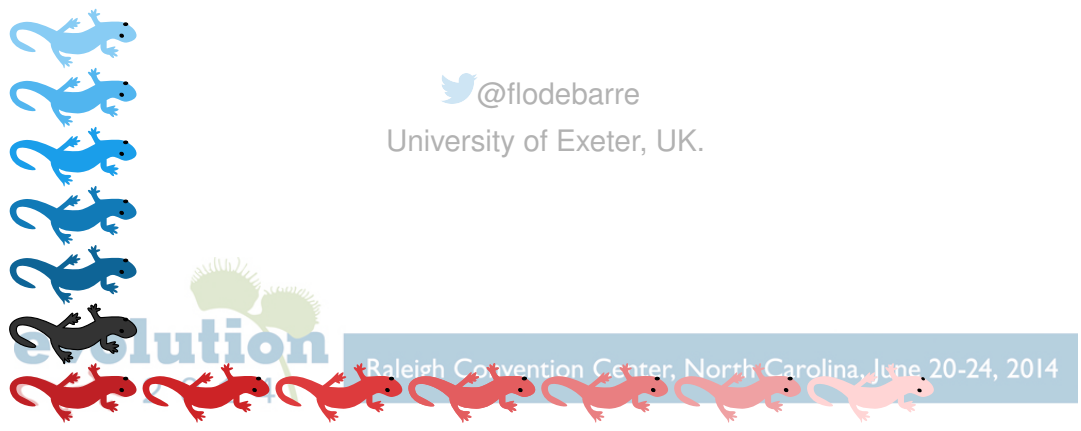




\section{Multidimensional (co)evolutionary stability}

Flo Débarre

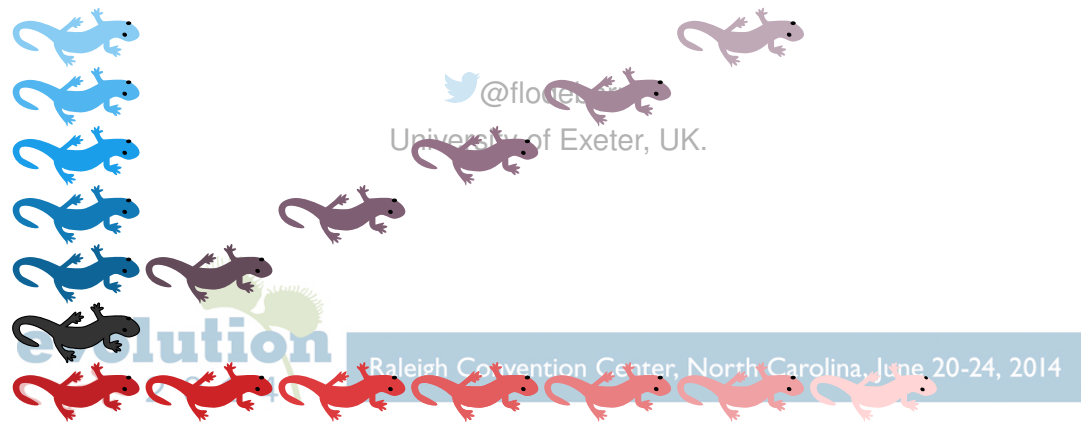




\section{Multiglingsion (co)evolutionary stability $C^{4}+0^{*}=$}

Flo Débarre

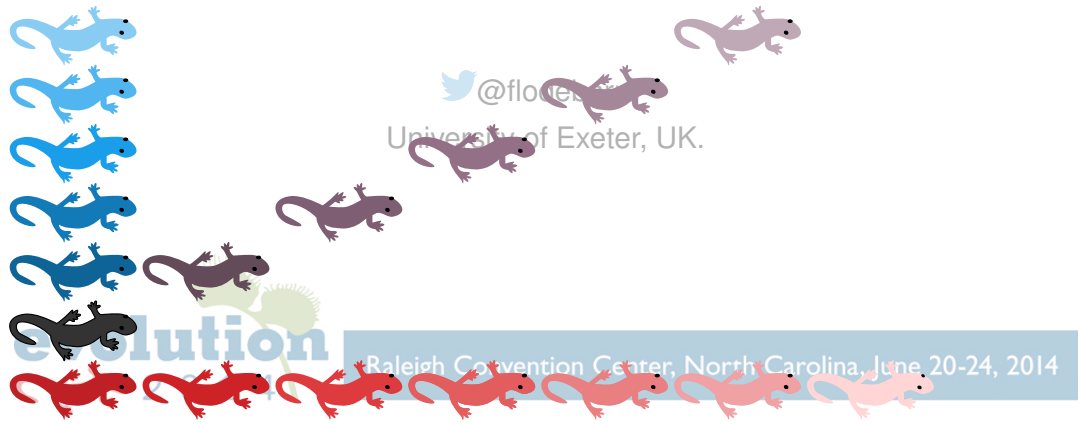




\section{Multiglingsion (co)evolutionary stability $C^{4}+0^{*}=$}

Flo Débarre

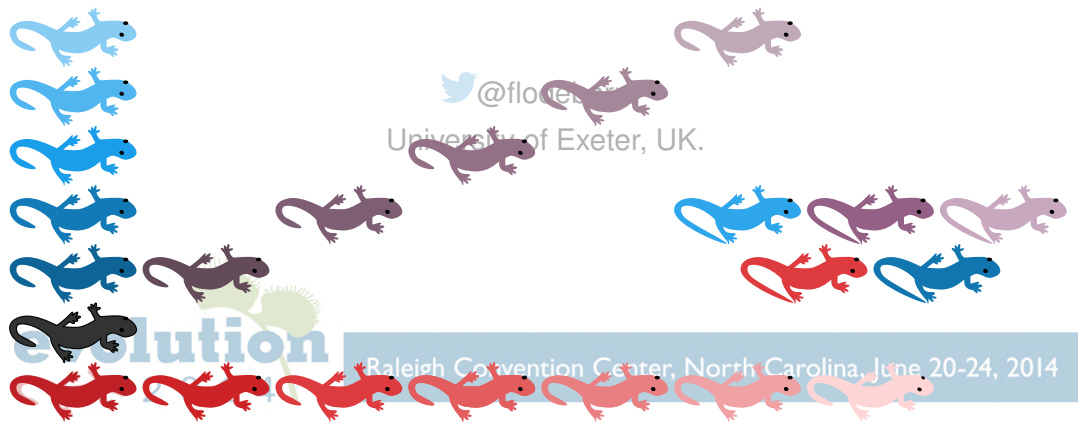



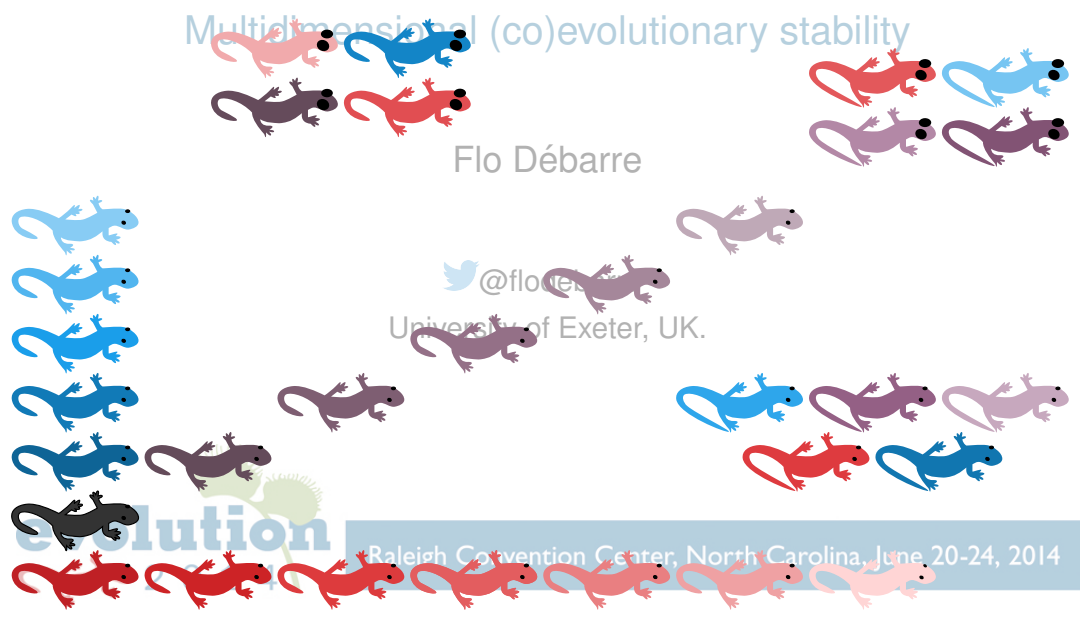


\section{Multiglin:}

Flo Débarre
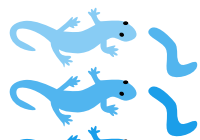

Q
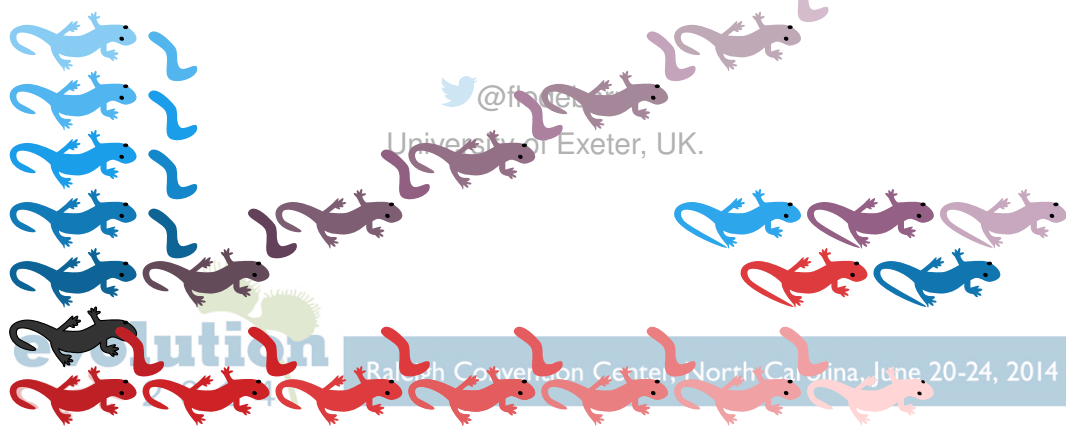


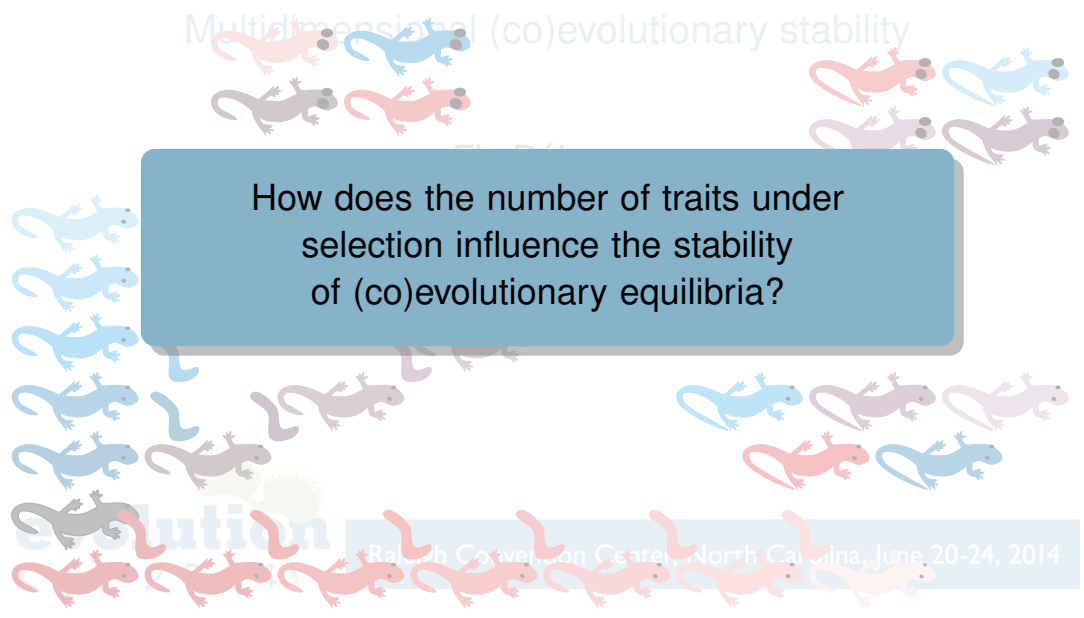




\section{Multiditases

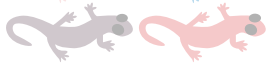

\section{How does the number of traits under selection influence the of (co)evolutionary equilibria?}




\section{Stabilities}

Convergence stability (CS)

Evolutionary stability (ES) 


\section{Stabilities}

Convergence stability (CS)

Changes in the mean traits Attractor/Repellor

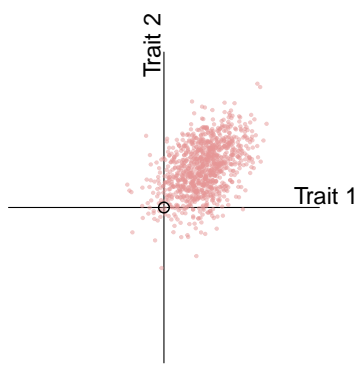




\section{Stabilities}

Convergence stability (CS)

Changes in the mean traits

Attractor/Repellor

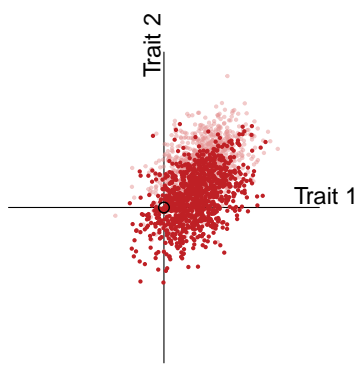




\section{Stabilities}

Convergence stability (CS)

Changes in the mean traits Attractor/Repellor

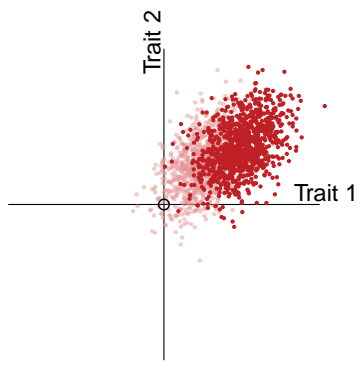




\section{Stabilities}

Convergence stability (CS)

Changes in the mean traits Attractor/Repellor

\section{Evolutionary stability (ES)}

Changes in the trait variances Stabilizing/Diversifying selection

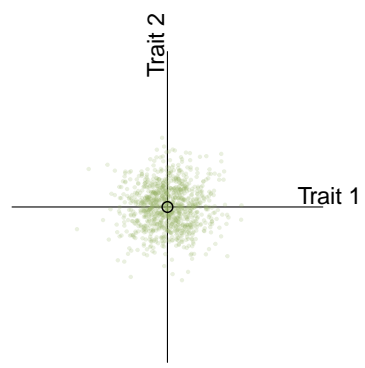




\section{Stabilities}

Convergence stability (CS)

Changes in the mean traits Attractor/Repellor

\section{Evolutionary stability (ES)}

Changes in the trait variances Stabilizing/Diversifying selection

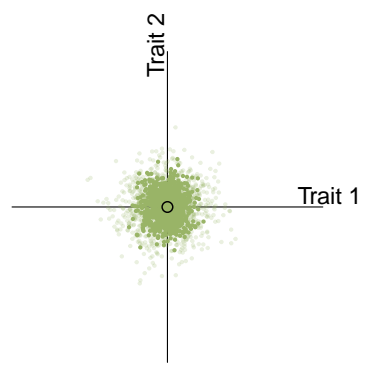




\section{Stabilities}

Convergence stability (CS)

Changes in the mean traits Attractor/Repellor

\section{Evolutionary stability (ES)}

Changes in the trait variances Stabilizing/Diversifying selection

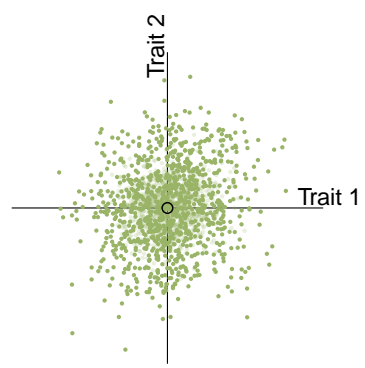


Three effects of dimensionality

- 2222 


\section{Three effects of dimensionality คै:}

Combinatorial effect

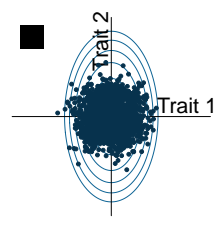




\section{Three effects of dimensionality}

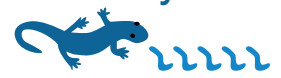

Combinatorial effect
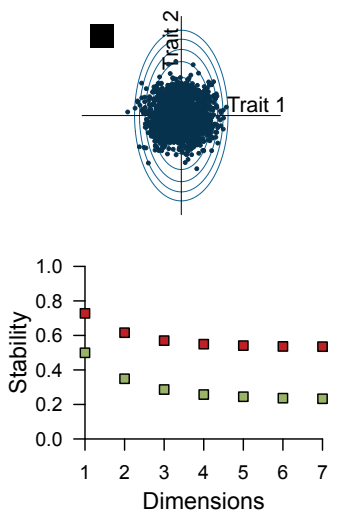

CS
ES 


\section{Three effects of dimensionality}

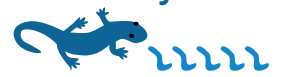

Combinatorial effect
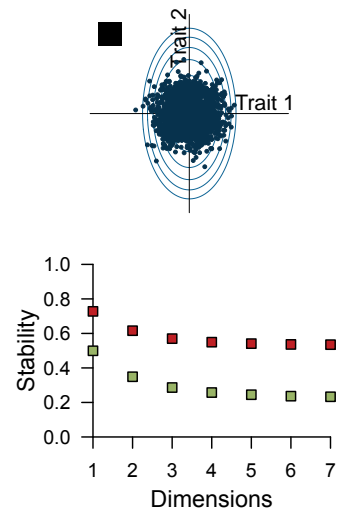

Epistatic interactions

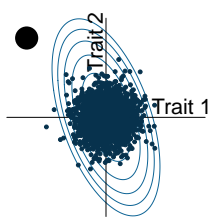

CS
ES 


\section{Three effects of dimensionality}

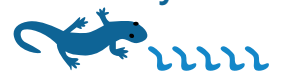

Combinatorial effect
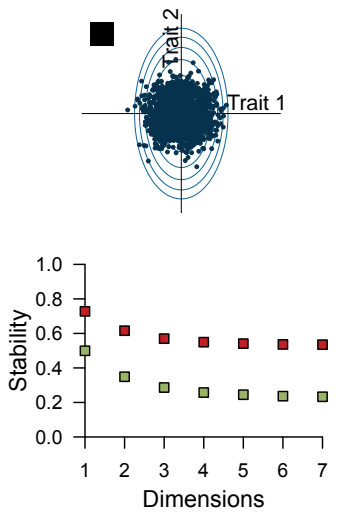

CS
ES
Epistatic interactions
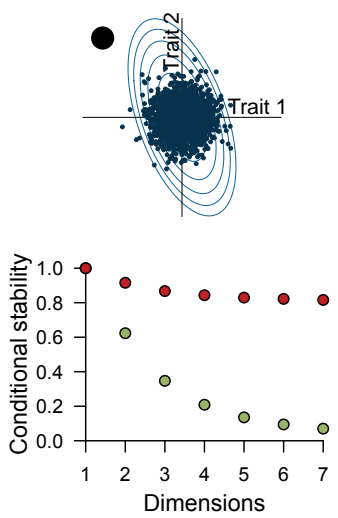

CS
ES $\boldsymbol{}$ 
Three effects of dimensionality

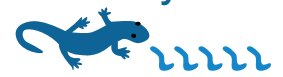

Combinatorial effect
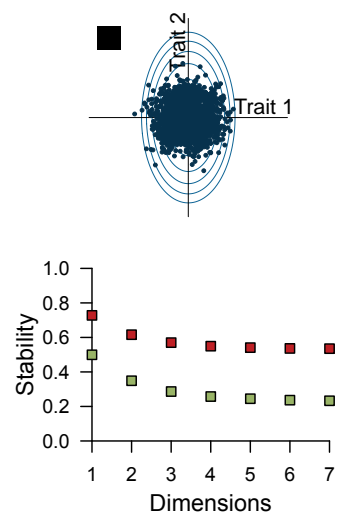

Epistatic interactions
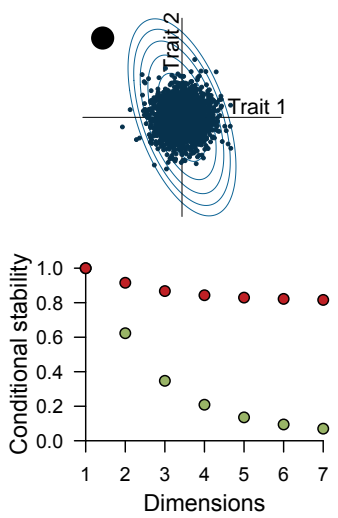

CS
ES
Trait correlations

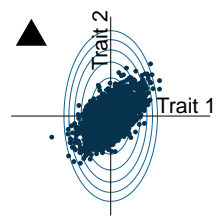


Three effects of dimensionality

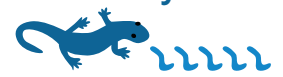

Combinatorial effect
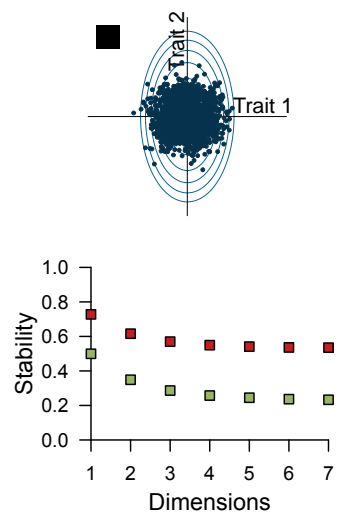

$\begin{array}{ll}C S & \checkmark \\ \text { ES } & \checkmark\end{array}$
Epistatic interactions
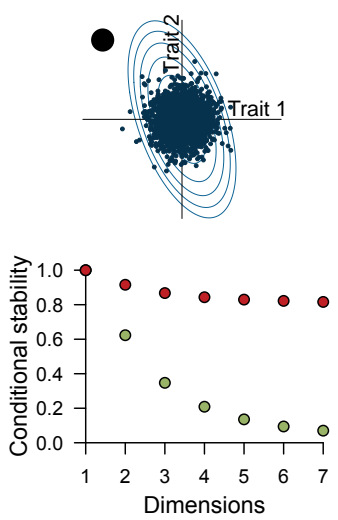

CS ES
Trait correlations
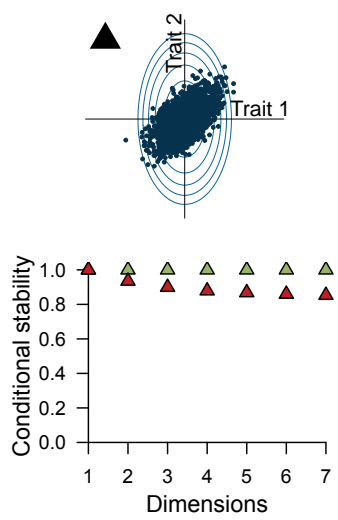

CS

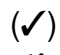
ES $x$ 


\section{Conclusion}

\section{Increased dimensionality destabilizes equilibria.}

Three effects of dimensionality:

Combinatorial effect Epistatic interactions Trait correlations

CS
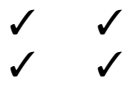

$(\sqrt{ }) \quad x$

For more details, see

Débarre, Nuismer \& Doebeli (2014), The American Naturalist, in press.

Talk uploaded on Figshare

Thanks for your attention!

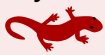

